\title{
The Use of Simulation Training to Accelerate the Rate of Forward Ice Skating Skill Acquisition
}

\author{
Nathan J Washington \\ School of Science and Health, Western Sydney University, PO box 2751, Sydney New South Wales, Australia \\ E-mail: n.washington@westernsydney.edu.au \\ Sera Dogramaci \\ New South Wales Institute of Sport, Figtree Drive, Olympic Park, Sydney, NSW, Australia \\ E-mail: sera.dogramaci@nswis,gov.au \\ Kylie A Steel (Corresponding author) \\ School of Science and Health, Western Sydney University, PO box 2751, Sydney New South Wales, Australia \\ E-mail: k.steel@westernsydney.edu.au \\ Eathan Ellem \\ School of Science and Health, Western Sydney University PO box 2751, Sydney New South Wales, Australia \\ E-mail: e.ellem@westernsydney.edu.au
}

Received: 08-02-2016

Accepted: 16-03-2016

Published: 30-04-2016

doi:10.7575/aiac.ijkss.v.4n.2p.11

URL: http://dx.doi.org/10.7575/aiac.ijkss.v.4n.2p.11

\begin{abstract}
Background: Australia's interest and participation in ice hockey is increasing, however a lack of access to facilities means familiarity with this sport is limited, and so too is the facilitation of skill development within an ecologically valid context. Objective: While numerous methods may be employed to address this, one resource which remains relatively unexplored is the StrideDeck Treadmill, therefore the purpose of this study was to investigate the effectiveness of this equipment with specific reference to the biomechanical changes for skating ability. Methods: $\mathrm{N}=$ 16 male athletes (Mage $=15.0 \pm 0.76$ yrs) from a junior league competition participated in this intervention based study. $\mathrm{n}=9$ were assigned to the training intervention (StrideDeck) once a week, while the control group $(\mathrm{n}=7)$ continued their normal training routines. Further, monthly sprint tests both on the StrideDeck and an on-ice protocol were conducted to track progress via kinematic analysis. Results: Data analysis revealed no significant overall effects for onice sprint skating performance after StrideDeck training; however there were significant kinematic differences between StrideDeck and ice conditions. Conclusions: Therefore while the StrideDeck may have merit in regard to physiological paramters, the results of this study do not support its use as a skill acquisition tool in regard to increasing skating ability.
\end{abstract}

Keywords: Simulation training, skill acquisition, treadmill, ice skating, ice hockey skating, ice skating stride

\section{Introduction}

Research has demonstrated the development of expertise in movement execution is influenced by factors such as feedback, instruction, and practice type (e.g., deliberate practice) (Ericsson \& Lehmann, 1996; Baker \& Young, 2014). Other factors that should also be considered are access to coaches, equipment, and facilities (Baker \& Young, 2014). However, in Australian contexts there are limitations to these resources; as such athletes must employ a variety of strategies and resources to accelerate their learning. For example, simulation training is a practice method designed to replicate the movements or environment associated with the context it is attempting to emulate (Cha et al., 2012). It is also a training method that is essential in cases where accessing specific environments is limited or there is a high risk of injury, thus impeding the mastery of a skill (Cha et al.2012).

Simulation training in sporting contexts helps athletes develop necessary skills that can be transferred into competition, thus improving the proficiency of skill execution and reducing error (de Groot et al., 2011). For example, ball projection machines replicate ball trajectories in sports such as cricket, volleyball, and tennis which can increase skill acquisition and execution (Pinder et al., 2011). Further, motor sport employs virtual simulators to enhance the driver's decision making skills, thus decreasing the risk of accidents and track hiring costs (de Groot et al., 2011). Such environmental constraints are evident within ice hockey, specifically the availability of ice rinks for skating practice. Australian ice hockey players lack access to a sufficient number of ice rinks which is disadvantageous for players. For example, Australia (ranked 36th) is limited to 10 indoor rinks for 4,264 players (426.4 players per rink) (International Ice Hockey Federation (IIHF), 2015), whereas Canada (ranked 1st) have 2,631 indoor and 5,000 outdoor rinks for 721,504 players (94.5 players per rink) (IIHF, 2015). Given restrictions in rink accessibility, Australian ice hockey players face different 
challenges compared to northern hemisphere (Soberlak \& Côté, 2003). For instance, skating in ice hockey is the most important movement skill when gauging success in competitive leagues (Bracko, 2004). Furthermore, talent scouts pay significant attention to skating when determining potential players for team selection (Hansen \& Reed, 1979). Ice hockey players need to be confident and powerful skaters in order to focus on strategy and tactics during high velocities in a game situation (Upjohn et al.2008); and maintain efficiency given the average skating distance is between $3 \mathrm{~km}$ $5 \mathrm{~km}$ per game (Montgomery et al., 2004). Therefore, the importance of skating combined with the lack of access to facilities to promote skating development reduces world ranking progression with top competitors such as Canada and United States of America.

Several simulation training methods currently exist that emulate ice surfaces, such as synthetic ice panels, slide boards, and skating treadmills, which provide glide-like properties to help enhance skating mechanics (Stidwill et al.,2010; Pies et al.,1998; Dreger, 1997; Nobes et al.2003). This allows skaters to replicate the movement patterns performed on ice in a different environment, thus continuing their skating development. Despite the prevalence of simulation tools and equipment focusing on the skating component, there is a lack of evidence observing the effectiveness of simulation training in ice hockey skating.

The current study utilises the StrideDeck Treadmill (SDT) (Figure 1.), which is a portable piece of equipment that focuses on the forward stride component of skating, which accounts for $85 \%$ of total skating time in ice hockey (Stamm, 2010). Therefore, the purpose of this study was twofold, firstly, to assess whether incorporating the SDT within regular training sessions increases skill acquisition of skating, and secondly whether any increases in performance translate to improve on-ice hockey sprint times.

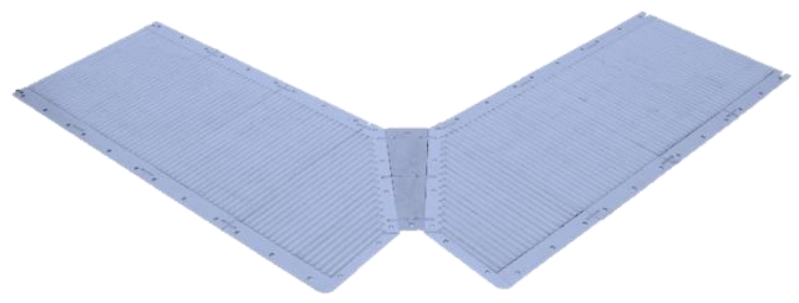

Figure 1. StrideDeck Treadmill

\section{Methods}

\subsection{Participants}

Sixteen male athletes $\left(M_{\text {age }}=15.0 \pm 0.76 \mathrm{yrs}, M_{\text {height }} 164.3 \pm 7.52 \mathrm{~cm}, M_{\text {weight }} 57.9 \pm 7.38 \mathrm{~kg}\right)$ from an Australian junior ice hockey team volunteered to participate in this study. Athletes varied in their level of ice hockey expertise (state representative $=6$, social $=10$ ), with $n=9$ and $\mathrm{n}=7$ players randomly allocated to training and control groups respectively. Each group contained a mix of attacking and defending players, however goaltenders were excluded from the study. Data collection for this study took place during the winter competition season (May to September) at the local ice rink participants used for training. Ethics approval was gained from Western Sydney University's Human Research Ethics committee, with athletes and their parent/guardians providing informed consent prior to participating in the study.

\subsection{Protocol}

The training group implemented the StrideDeck Treadmill (SDT) prior to each on ice practice session, while the control group continued their regular practice. To test if the SDT had any effect on skating performance athletes underwent on ice sprint tests every four weeks (four sprinting tests took place over the course of the study) to track progress and observe any improvements that may have occurred. Further, to determine if the SDT is a viable skating training apparatus, a biomechanical comparison was implemented to assess kinematic differences between strides in SDT and sprint test protocols.

Participants wore their full on-ice equipment during SDT training sessions, including skates, helmets, with hockey stick in hand (Figure 2). Rubber skate guards were worn on the SDT to protect the skate blades as the plastic rollers create friction resulting in an increase in wear. Prior to SDT sessions, athletes completed a familiarisation period with the treadmill which involved performing 10 consecutive strides. During SDT sessions, athletes engaged in forward stride motion for two minutes, this meant athletes maintained a consistent pace with full leg extensions.

Athletes then increased the extension rate to a higher intensity for an additional minute, resulting in a total intervention time of three minutes. Training and control groups completed four on-ice sprint tests throughout the season. Baseline forward straight line sprinting time was based on the International Ice Hockey Federation Skills Challenge Manual (IIHF, 2015). Participants were required to sprint as fast as possible from the starting line to the finishing line (100ft/30.48m distance).The forward sprint was filmed using a tripod mounted Sony High Definition video camera recording 50fps (Sony Corporation, Tokyo, Japan) allowing a biomechanical comparison between on-ice and SDT to take place. The forward stride consists of three phases, beginning when the blade has made initial contact with the ice, 
progressing through the glide, push-off, and recovery phases (De Boer et al., 1986). Timing commenced as soon as the participant initiated movement from the starting line until their torso crossed the finish line. The result of the two trials of the forward sprint were recorded, then averaged to produce a mean time for forward skating.

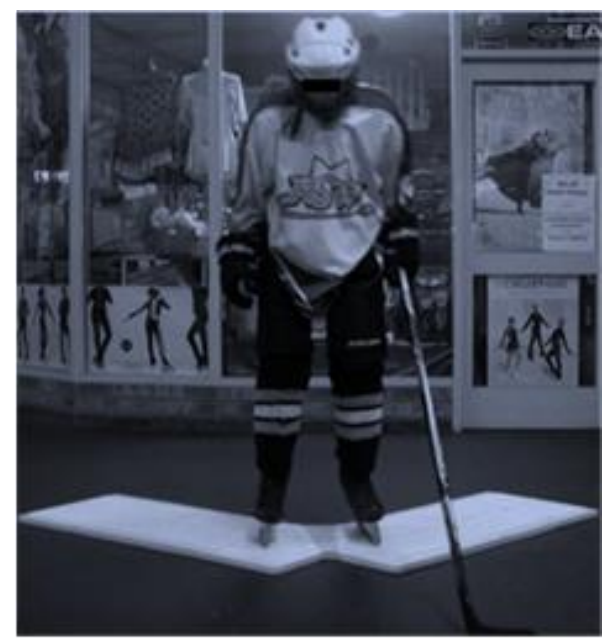

Figure 2. Subject on StrideDeck Treadmill with full equipment as well as skate guards

\subsection{Analysis}

A biomechanical comparison between on-ice and SDT was completed using Kinovea software (version 0.8.15, Kinovea, France). This additional analysis was employed to determine whether the stride leg angles performed on the SDT were similar to the sprint testing protocol. Further, analysis measured stride kinematics across four SDT sessions and sprint tests to measure if any changes occurred over time.

Using the Kinovea software, common landmarks were measured for both legs, specifically leg adduction (medial), leg abduction (lateral), and ankle flexion angles (Figure 3). Measurements were taken when the athletes were striding at a high intensity in the final minute on the SDT.

Statistical analysis was conducted SPSS (version 22.0) to assess whether significant changes occurred in skating sprint performance after SDT sessions when compared to the control group. A linear mixed model was applied for the dependant variable of forward sprint time (FWD). Fixed factors were group (Training and Control) and session (pre/post ice), with participant being a random factor. Further, the dependant variables for biomechanical analysis were medial, lateral, and ankle flexion angles. Fixed factors were leg (left and right) and session (pre/post ice and SDT). Moreover, a final analysis was conducted that determined whether significant changes occurred in SDT performance only. Four sessions were analysed in total, with analysis focusing on the above kinematic dependant variables in each session (SDT1, SDT2, SDT3, SDT4). An alpha level of $p=<.05$ was set as the criterion for significance for all statistical procedures.

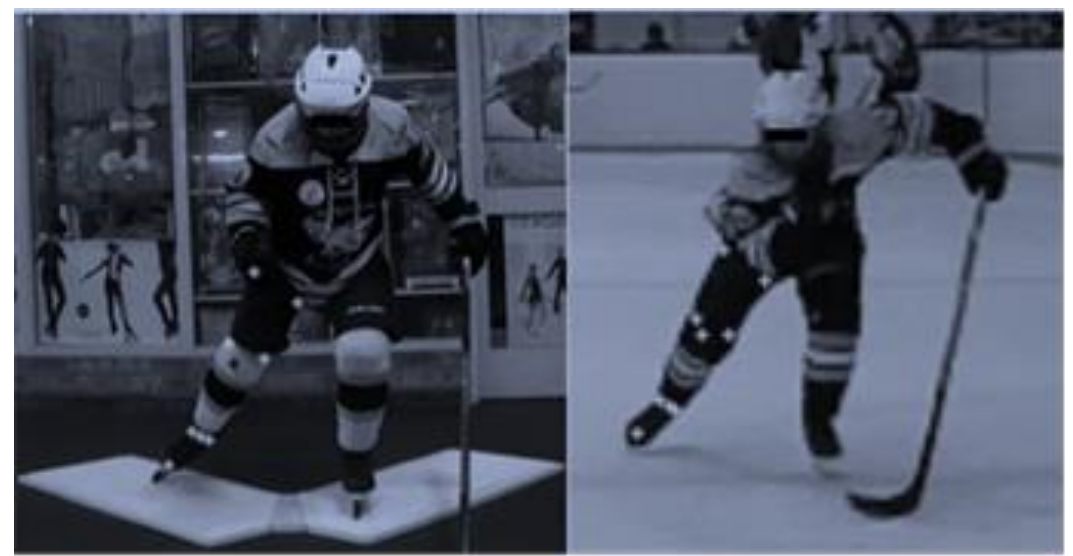

Figure 3. Kinematic markers identify the movements analysed. Medial/ leg adduction, lateral/leg abduction, and foot/ ankle

\section{Results}

\subsection{Sprint Test}

Analysis of sprint times returned no significant main effects for Group $F(1,15)=1.16, p=.299$ or session $F(3,29)=$ $0.54, p=.659$, nor was there a significant Group x Session interaction $F(3,29)=1.12, p=.359$. While training and control groups returned differences between pre- and post-tests, these times were not significant (Table 1). 
Table 1. Differences between the training and control groups based on session

\begin{tabular}{lll}
\hline & Group & \\
\cline { 2 - 3 } Session & Training & Control \\
\hline Pre-Test & $5.22(0.41)$ & $5.58(0.39)$ \\
Post-Test & $5.24(0.39)$ & $5.41(0.58)$ \\
\hline
\end{tabular}

Note. Mean sprint time for training and control groups in pre- and post-tests when groups were combined, data presented as mean time (standard deviation).

\subsection{Kinematic comparison between ice and SDT}

Analysis of medial kinematic data returned a significant overall effect for Session $F(3,462)=36.06, p=<.001$ (Figure 4 ), as well as a significant Session $\mathrm{x}$ Leg interaction $F(3,461)=9.45, p=<.001$. When legs were combined, there were significant differences in average medial angles between ice and SDT sessions (Table 2). Further, the significant interaction is attributable to differences between left and right legs in pre-ice, as well as post-deck sessions (Table 3).

\section{Leg abduciton data}

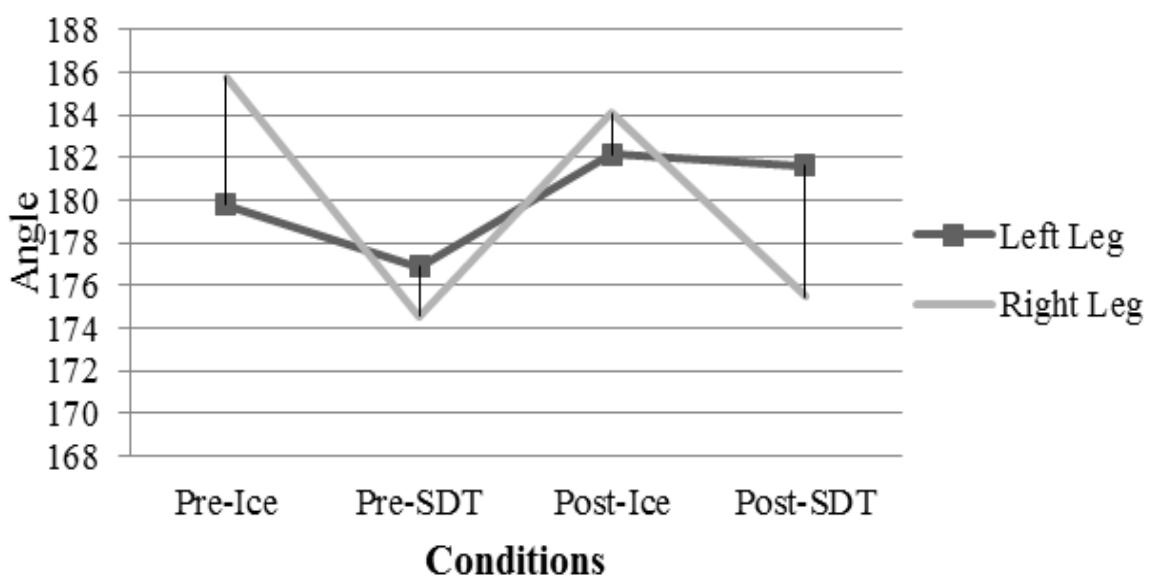

Figure 4. Medial kinematic angle differences between legs on different conditions

Table 2. Kinematic data based on session (Pre-Post) and condition (Ice vs SDT)

\begin{tabular}{llll}
\hline & Angle & & Ankle \\
\cline { 2 - 4 } Session & Medial & Lateral & $150.98(5.80)_{1,2,3}$ \\
\hline Pre-Ice & $174.79(5.17)_{1,2}$ & $182.78(4.85)_{1,2}$ & $147.05(5.37)_{2,4,6}$ \\
Post-Ice & $174.69(4.92)_{3,5}$ & $183.12(4.48)_{3,5}$ & $161.76(5.53)_{1,4,5}$ \\
\hline Pre-Deck & $183.48(5.013)_{1,3,4}$ & $175.71(4.62)_{1,3,4}$ & $156.89(5.53)_{3,5,6}$ \\
Post-Deck & $179.01(5.013)_{2,4,5}$ & $178.54(4.62)_{2,4,5}$ & \\
\hline
\end{tabular}

Note. Summary of kinematic variables across ice and StrideDeck sessions with legs combined, subscript numbers indicate significance between sessions for a given variable only, not between variables, data presented as mean angle (standard deviation).

Table 3. Presents data demonstrating session and leg interaction.

\begin{tabular}{lllll}
\hline \multirow{2}{*}{ Session } & Leg & Angle & & \\
\cline { 3 - 5 } Pre-Ice & L & Medial & Lateral & Ankle \\
& $\mathrm{R}$ & $177.14(5.69)$ & $179.76(5.60)$ & $155.17(6.66)$ \\
\multirow{2}{*}{ Post-Ice } & $\mathrm{L}$ & $172.43(5.69)$ & $185.80(5.60)$ & $146.78(6.66)$ \\
& $\mathrm{R}$ & $175.34(5.07)$ & $182.15(4.71)$ & $148.84(5.64)$ \\
\multirow{2}{*}{ Pre-Deck } & $\mathrm{L}$ & $174.03(5.43)$ & $184.10(5.24)$ & $145.25(6.24)$ \\
\multirow{2}{*}{ Post-Deck } & $\mathrm{R}$ & $182.30(5.43)$ & $176.89(5.24)$ & $163.05(6.24)$ \\
& $\mathrm{L}$ & $184.65(5.43)$ & $174.54(5.24)$ & $160.48(6.24)$ \\
\hline
\end{tabular}

Note. Summary of Session x Leg kinematic variables, data presented as mean angle (standard deviation). 
Analysis of lateral kinematic data returned a significant overall effect for Session $F(3,462)=18.88, p=<.001$, as well as a significant Session $\mathrm{x}$ Leg interaction $F(3,461)=9.39, p=<.001$ (Table 3). When legs were combined, there were again significant differences in average lateral angles between ice and SDT sessions (Table 2).

Analysis of ankle kinematic data returned a significant overall effect for Session $F(3,462)=49.27, p=<.001$, $\operatorname{Leg} F(1$, $460)=8.77, p=.003$ (Figure 5), as well as a significant Session $\mathrm{x}$ Leg interaction $F(3,461)=5.39, p=<.001$ (Table 3). When legs were combined, again there were significant differences in ankle angles between ice and SDT sessions (Table 2). When sessions were combined, there was a greater angle in the right $(M=155.60, S D=5.14)$ compared to the left ankle $(M=152.74, S D=5.18)$.

3.3 StrideDeck session kinematic analysis

Analysis of medial kinematic SDT data returned significant overall effects for Session $F(3,385)=11.65, p=.005$ and $\operatorname{Leg} F(1,385)=50.90, p=<.001$, as well as a significant Session x Leg interaction $F(3,385)=5.62, p=<.001$. Further, analysis of lateral kinematic SDT data returned significant overall effects for Session $F(3,385)=4.79, p=.003$ and $\operatorname{Leg} F(1,385)=27.57, p=<.001$, as well as a significant Session x Leg interaction $F(3,385)=2.69, p=.046$. Moreover, analysis of ankle kinematic SDT data returned significant overall effects for Session $F(3,385)=8.05, p=<$ .001 , as well as a significant Session $\mathrm{x}$ Leg interaction $F(3,385)=4.98, p=.002$ (Figure 6).

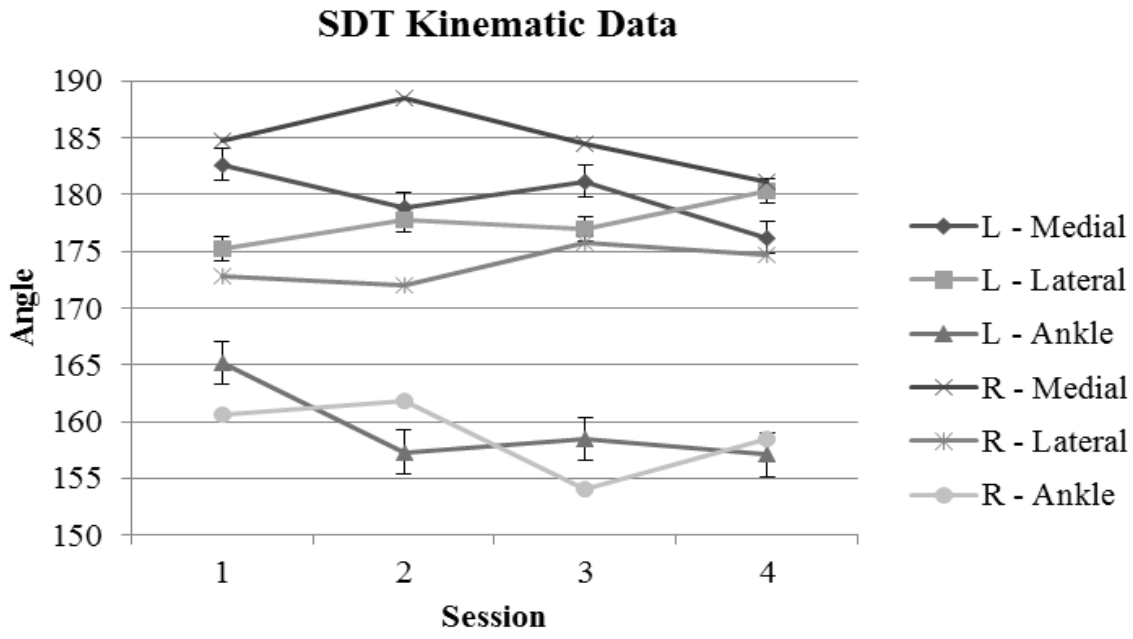

Figure 5. Ankle kinematic angle differences of legs on different conditions.

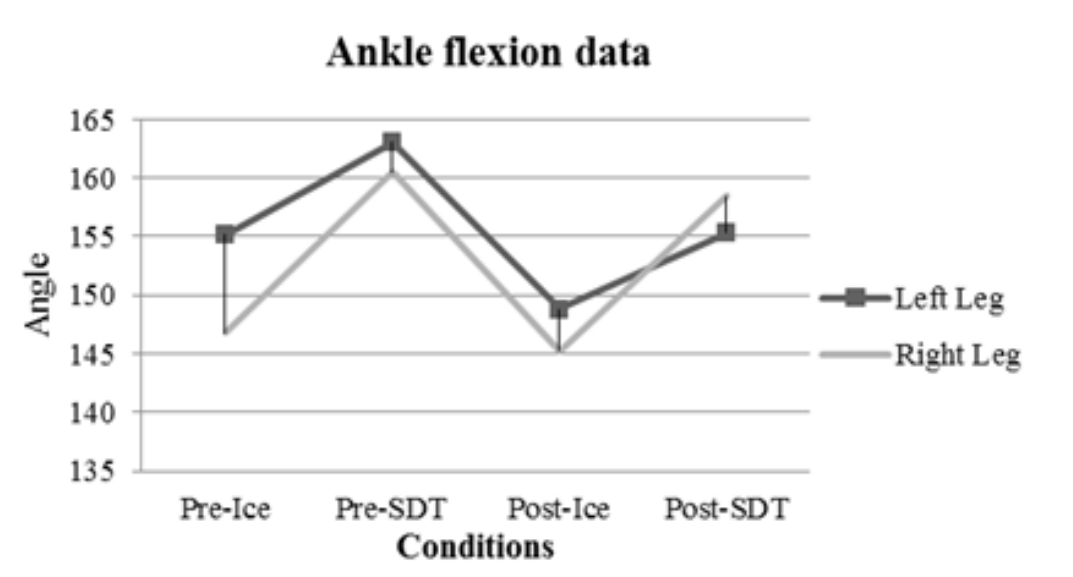

Figure 6. SDT kinematic data only for all angle variables across four sessions.

\section{Discussion}

The aim of this study was to assess whether the StrideDeck Treadmill (SDT) improved ice skating performance using a pre-post intervention design. The outcome was measured based on improvements in forward ice skating sprint times. While there were no significant improvements in skating performance between pre- and post- sprint tests, there were kinematic trends for post-SDT and post-ice conditions. However, this demonstrated the SDT did not sufficiently contribute to enhanced sprint skating ability.

While there were no significant findings for sprint time, there were significant kinematic differences between medial and lateral angles for all sessions when comparing skating surfaces. This demonstrated movement patterns on the SDT did not reflect that of skating on ice; this may be due to the changes in mechanics associated with high skating velocities. Marino (1977) found while athletes are skating in sprint intensity, they spend more time in single support leg phase than double support leg phase. This was evident in the sprinting kinematic of the current study when measuring 
the push-off leg angles on the ice. The athletes opposing leg would be in the recovery phase whereas on the SDT the opposite leg that was being measured was positioned on the ground to balance the skater. However, the length of time spent in double support phase was not measured here; therefore it is unclear whether this affected results.

In addition to the significant findings for medial and lateral angles, ankle flexion angles were significantly different between the SDT and ice skating. Participants wore rubber skate guards to protect the blades of the boot when performing on the SDT; resulting in an increase in friction between the guards and the rollers. The increased resistance may have disrupted the athlete's movement pattern, resulting in a greater level of self-organisation to overcome the resistance, which also affected and led to significant differences in medial and lateral angles (Magill, 2010). Similar movement pattern disruption was also found due to the disparity in the surface coefficient of friction in a study carried out by Stidwill et al., (2010). The subjects had a more upright trunk angle with increased stride frequency on synthetic ice surfaces compared to ice conditions. Although these were minor differences in the study (Stidwill et al. 2010) this demonstrated the effect resistance can have on the movement pattern when executed on frictionless surfaces (i.e., ice). High velocity speeds play a significant role in increasing joint range of motion, with Lafontaine (2007) and Buckeridge et al., (2015) suggesting joint motion amplitude is proportional to increases in velocity. Further, Chang et al., (2009) as well as De Koning et al., (1995) found skaters had to accommodate high skating speed with increased hip abduction angles and rate. Without the extension rate demonstrating the intensity between the two surfaces, the range of motion is compromised on the SDT, therefore resulting in significant differences across all measurements in comparison to ice conditions (Table 2.).

The glide phase contributes to the velocity of ice skating, with the gliding motion utilising the stretch shortening cycle (Upjohn et al., 2008). This is executed with a forward trunk lean which applies more weight on the gliding skate. By increasing knee flexion (eccentric contraction) a player can use the elastic stored energy to apply more force in the push-off (concentric contraction) resulting with an increased stride length (Upjohn et al., 2008; Stamm, 2010). The design of the SDT did not allow a glade phase to occur, this further illustrates why there was a lack of kinematic differences between the SDT and on-ice.

Kinematic measurements could be more reliable in future studies by employing kinematic markers placed on the skaters to increase the accuracy of estimations in analysis. Moreover, measurements were only taken from the frontal plane; whereas additional data could be collected from the sagittal plane, thus providing insight to stride length and analysis of the recovery phase. Despite study measurement limitations, supported literature demonstrates that skating mechanics on both SDT and ice are significantly different, and these findings are supported by previous ice skating biomechanical studies (De Boer et al. 1986; Marino, 1977).

When considering motor control, Maslovat et al., (2004) suggest practice conditions should match test conditions as closely as possible, thus leading to an increase in performance in the latter. This is true in simulation training studies where transfer of skill acquisition from a training environment to real environments are evident due to the familiarity in neuromotor processes, decision-making, and correct skill execution volume (de Groot et al., 2011; Pinder et al.,2011; Del Sal et al.2009; Willaert et al., 2012; Bekker \& Lotz, 2009). However, this did not occur in the current study as there were significant differences in movement patterns between SDT and ice. The design of the SDT may not have allowed participants to perform normal skating movement patterns. Athletes are able to perform the gliding phase and manoeuvres on ice, whereas this is impossible on the SDT. Practicing incorrect movement patterns can inhibit the motor skill which enforces why the SDT had produced no transfer of skills onto real environments (Kottke et al. 1978).

It should be noted that adequate time is needed for subjects to become familiarised with an apparatus otherwise testing performance may not be representative of their ability (Lockwood \& Frost, 2007). Lockwood and Frost (2007) found that it had taken subjects up to six weeks to become familiar and skate efficiently on the skating treadmill. Considering skaters in this study were only exposed to the SDT once a week for a period of four weeks, this may not have afforded enough time for subjects to become familiar with the device.

\section{Conclusion}

Overall, the SDT resulted in significant biomechanical differences, e.g., differences in leg abduction, adduction, and ankle flexion when compared to ice conditions; however this did not translate to improved sprint ability on ice. It is likely the motor patterns for the SDT compare to on-ice sprinting are dissimilar, thus the associated mechanics for each surface do not correlate with each other. We therefore suggest that the SDT in its current form does not provide suitable platform for skill development, though may be useful for improved fitness. Future studies could possibly assess whether the SDT can be utilised as a fitness method to increased skating endurance.

\section{Acknowledgment}

We are thankful to the Canterbury ice hockey team for volunteering their time to participate. We are also grateful for the New South Wales Institute of Sport for providing the equipment and supporting our study.

\section{References}

Baker, J., \& Young, B. (2014). 20 years later: Deliberate practice and the development of expertise in sport. International Review of Sport and Exercise Psychology, 7(1), 135-157.

Bekker, J., \& Lotz, W. (2009). Planning formula one race strategies using discrete event simulation. The Journal of the Operational Research Society, 60(7), 952-961.

Bracko, R. (2004). Biomechanics powers ice hockey performance. Biomechanics, 47-53. 
Buckeridge, E., LeVangie, M., Stetter, B., Nigg, S., \& Nigg, B. (2015). An on-ice measurement approach to analyse the biomechanics of ice hockey skating. PLOS ONE, 10(5).

Cha, M., Han, S., Lee, J., \& Choi, B. (2012). A virtual reality based fire training simulator integrated with fire dynamics data. Fire Safety Journal, 50, 12-24.

Chang, R., Turcotte, R., \& Pearsall, D. (2009). Hip adductor muscle function in forward skating. Sports Biomechanics, $8(3), 212-222$.

De Boer, R., Schermerhorn, P., Gademan, J., de Groot, G., \& van Ingen Schenau, G.J. (1986). Characteristic stroke mechanics of elite and trained male speed skaters. International Journal Sport Biomechanics, 2, 175-185.

Del Sal, M., Barbieri, E., Garbati, P., Sisti, D., Rocchi, M., \& Stocchi, V. (2009). Physiologic responses of firefighter recruits during a supervised live-fire work performance test. Journal of Strength and Conditioning Research, 23(8), 2396-2404.

de Groot, S., Mulder, M., \& Wieringa, P. (2011). Car racing in a simulator: Validation and assessment of brake pedal stiffness. Teleoperators \& Virtual Environment, 20(1), 47-61.

De Koning, J., Thomas, R., Berger, M., de Groot, G., \& van Ingen Schenau, G.J. (1995). The start in speed skating: From running to gliding. Medicine and Science in Sports and Exercise, 27, 1703-1708

Dreger, R. (1997). Using skate-treadmills to train hockey players for speed. Strength \& Conditioning Journal, 19(6), 33-35.

Ericsson, K., \& Lehmann, A. (1996). Expert and exceptional performance: Evidence of maximal adaptation to task constraints. Annual Review of Psychology, 47(1), 273-305.

Hansen, H., \& Reed, A. (1979). Functions and on-ice competencies of a high caliber hockey player-a job analysis. In Science in Skiing, Skating, and Hockey. Proceedings of the International Symposium of Biomechanics in Sports (pp. 107-115). Del Mar, CA: Academic Publishers.

International Ice Hockey Federation (IIHF), (2015), Country profiles. Retrieved from: http://www.iihf.com/iihfhome/countries/australia/

International Ice Hockey Federation (IIHF), (2015). Skills manual challenge: Test 3. Retrieved from: http://www.ihi.is/gogn/skill_challange.pdf

Kottke, F., Halpern, D., Easton, J., Ozel, A., \& Burrill, C. (1978). The training of coordination. Archives of Physical Medicine Rehabilitation, 59, 567-572.

Lafontaine, D. (2007). Three-dimensional kinematics of the knee and ankle joints for three consecutive push-offs during ice hockey skating starts. Sports Biomechanics, 6(3), 391-406.

Lockwood, K., \& Frost, G. (2007). Habituation of 10-year-old hockey players to treadmill skating, Sports Biomechanics, 6(2), 145-154.

Magill, R. (2010). Motor Learning and Control: Concepts and Applications (9th ed.). New York: McGraw Hill-Int.

Marino, G. (1977). Kinematics of ice skating at different velocities. American Alliance for Health, Physical Education and Recreation, 48(1), 93-97.

Maslovat, D., Chus, R., Lee, T., \& Franks, I. (2004). Contextual interference: Single task versus multi-task learning. Motor Control-Champaign, 8(2), 213-233.

Montgomery, D., Nobes, K., Pearsall, D.J., \& Turcotte, R. (2004). Task analysis (hitting, shooting, passing and skating) of professional hockey players. In D.J Pearsall and A.B Ashare (Ed.), Safety in Ice Hockey: Fourth Volume (pp. 288295). West Conshohocken, PA: ASTM International.

Nobes, K., Montgomery, D., Pearsall, D. J., Turcotte, R. A., Lefebvre, R., \& Whittom, F. (2003). A comparison of skating economy and on-ice and on the skating treadmill. Canadian Journal of Applied Physiology, 28(1), 1-11.

Pies, N., Provost-Craig, M., Neeves, R., \& Richards, J. (1998). Cardiopulmonary responses to slideboard exercise in competitive female ice skaters. Journal of Strength and Conditioning Research, 12(1), 7-11.

Pinder, R., Renshaw, I., Davids, K., \& Kerhervé, H. (2011). Principles for the use of ball projection machines in elite and developmental sport programmes. Sports Medicine, 41(10), 793-800.

Stamm, L. (2010). Laura Stamm's Power skating (4th ed.). United States: Human Kinetics.

Stidwill, T., Pearsall, D. J., \& Turcotte, R. A. (2010). Comparison of skating kinetics and kinematics on ice and on a synthetic surface. Sports Biomechanics, 9(1), 57-64.

Soberlak, P., \& Côté, J. (2003). The developmental activities of elite ice-hockey players. Journal of Applied Sport Psychology, 15, 41-49.

UpJohn, T., Turcotte, R., Pearsall, D., \& Loh, J. (2008). Three-dimensional kinematics of the lower limbs during forward ice hockey skating. Sports Biomechanics, 7(2), 206-221.

Willaert, W., Aggarwal, R., Herzeele, I., Cheshire, N., \& Vermassen, F. (2012). Recent advancements in medical simulation: Patient-specific virtual reality simulation. World Journal of Surgery, 36(7), 1703-1712. 